\title{
Resveratrol improves prostate fibrosis during progression of urinary dysfunction in chronic prostatitis by mast cell suppression
}

\author{
HUIZHI ZENG ${ }^{1}$, YI HE ${ }^{2}$, YANG YU ${ }^{2}$, JIASHU ZHANG ${ }^{3}$, XIAONA ZENG $^{3}$, \\ FENGTAO GONG ${ }^{3}$, QI LIU ${ }^{3}$ and BO YANG ${ }^{2}$
}

\author{
Departments of ${ }^{1}$ General Surgery and ${ }^{2}$ Urology, The Second Affiliated Hospital of Dalian Medical University; \\ ${ }^{3}$ College of Pharmacy, Dalian Medical University, Dalian, Liaoning 116044, P.R. China
}

Received March 23, 2017; Accepted September 14, 2017

DOI: $10.3892 / \mathrm{mmr} .2017 .7960$

\begin{abstract}
Voiding dysfunction is the primary clinical manifestation of chronic prostatitis (CP), which is a common urological disease. The present study investigated whether prostate fibrosis was associated with urinary dysfunction in $\mathrm{CP}$ and if resveratrol improved urinary dysfunction, and the underlying molecular mechanism. A rat model of $\mathrm{CP}$ was established via subcutaneous injections of the pertussis-diphtheria-tetanus vaccine, which was followed by treatment with resveratrol. Bladder pressure and volume tests were performed to investigate the effect of resveratrol on urinary dysfunction in CP rats. Western blotting and immunohistochemical staining examined the expression levels of tryptase, chymase, transforming growth factor (TGF)- $\beta$, Wnt and $\alpha$-smooth muscle actin ( $\alpha$-SMA). The results demonstrated that the maximum capacity of the bladder, residual urine volume and maximum voiding pressure were increased significantly in the $\mathrm{CP}$ group compared with the control group. Mast cell (MC) activation, the activity of TGF- $\beta /$ Wnt $/ \beta$-catenin pathways, and the expression levels of tryptase and $\alpha$-SMA in the CP group were increased significantly compared with the control group. Resveratrol treatment significantly reversed these factors. Therefore, the results indicate that MC infiltration may induce prostate fibrosis, which exhibits a close association with urinary dysfunction in CP. Resveratrol may improve fibrosis via the suppression of $\mathrm{MC}$ activation and TGF- $\beta / \mathrm{Wnt} / \beta$-catenin pathway activities.
\end{abstract}

Correspondence to: Dr Qi Liu, College of Pharmacy, Dalian Medical University, 9 Western Section, Lvshun South Street, Lvshunkou, Dalian, Liaoning 116044, P.R. China

E-mail: 18842609110@139.com

Professor Bo Yang, Department of Urology, The Second Affiliated Hospital of Dalian Medical University, 467 Zhongshan Road, Shahekou, Dalian, Liaoning 116044, P.R. China

E-mail: yangbo20160101@163.com

Key words: chronic prostatitis, urinary dysfunction, mast cells, fibrosis, resveratrol

\section{Introduction}

Chronic prostatitis (CP) is a common disease in urology, and voiding dysfunction is the primary clinical manifestation of CP (1), which affects the quality of life of patients with CP. Urinary tract obstruction was previously reported in patients with $\mathrm{CP}$ upon urodynamic examination (2). Prostate fibrosis is a contributing factor to lower urinary tract symptom (LUTS) etiology (3).

Tissue fibrosis is reported to be associated with tissue damage caused by various factors, including aging (4), infection (5), tumors (6) and additional secondary diseases in various organs, which leads to dysfunction. The mechanism of fibrosis involves the accumulation of myofibroblasts, collagen deposition, remodeling of the extracellular matrix and elevated tissue rigidity. High levels of collagen in tissue leads to organ dysfunction as tissue elasticity and compliance are reduced $(4,5)$. However, the role of fibrosis in the development of $\mathrm{CP}$ is poorly understood. Therefore, the present study investigated whether $\mathrm{CP}$ with urinary dysfunction was associated with collagen accumulation in the prostate.

Mast cells (MCs) are important effector cells in tissue fibrosis $(7,8)$. MC activation and degranulation leads to the release of inflammatory and profibrotic mediators, which promotes tissue fibrosis. MCs express serine proteases, tryptase and chymase, which are associated with fibrosis in certain diseases $(9,10)$. However, the involvement of these proteases in $\mathrm{CP}$ is poorly understood. Therefore, the role of tryptase and chymase in the pathogenesis of $\mathrm{CP}$ was investigated in the present study. The pleiotropic cytokine, transforming growth factor- $\beta$ (TGF- $\beta$ ), serves numerous physiological roles in vivo, including the initiation and control of fibrosis (11). The profibrotic effects of MCs are closely associated with TGF- $\beta$ (12). Experimental studies have associated increased MC numbers and staining intensity with increased TGF- $\beta$ production and interstitial fibrosis $(12,13)$. The $\mathrm{Wnt} / \beta$-catenin signaling pathway has a vital role in TGF- $\beta$-induced fibrosis (14). Therefore, the potential role of the Wnt $/ \beta$-catenin/TGF- $\beta$ signaling pathway in the pathogenesis of $\mathrm{CP}$ was also determined in the present study.

Resveratrol (15) (trans-3,4,5-trihydroxy stilbene) is present in various plants and foods, including Cassia, pine trees, grapes, wine, mulberry and peanuts. Resveratrol was originally used as 
a phytoalexin (16), and the reliable and extensive anti-inflammatory effects of resveratrol gained the attention of researchers (17). Resveratrol treatment represses and reverses prostate fibroblast to myofibroblast phenoconversion in vitro (18). Our previous studies identified that resveratrol can improve the outcome of prostatitis $(19,20)$. However, to the best of our knowledge, no reports have investigated the effect of resveratrol on mast cell induced fibrosis in prostatitis. Antifibrotic therapeutics may be efficacious for the treatment of LUTS (18).

Over the past decades, a variety of animal models of prostatitis have been established with the aim of deciphering the pathogenesis of CP. The present study employed a rat model of experimental autoimmune prostatitis, which has been widely used $(21,22)$. This model exhibits the majority of the characteristics of human chronic autoimmune prostatitis, as specific lymphocytes against prostate antigens and histological lesions are observed in the prostate (23).

The present study investigated whether prostate fibrosis was associated with urinary dysfunction in $\mathrm{CP}$ and whether resveratrol improved urinary dysfunction. The activity of the c-kit/stem cell factor (SCF) and TGF- $\beta / \mathrm{Wnt} / \beta$-catenin signaling pathways, the number of $\mathrm{MC}$ infiltrates and the expression levels of tryptase, chymase and $\alpha$-smooth muscle actin ( $\alpha$-SMA) in CP rats was investigated.

\section{Materials and methods}

Chemicals. Resveratrol of $>99 \%$ purity was purchased from Dalian Meilun Biotech Co., Ltd. (Dalian, China). The pertussis-diphtheria-tetanus (DPT) vaccine was obtained from the Wuhan Institute of Biological Products Co., Ltd. (Wuhan, China). All other chemicals used in the present study were of analytical grade and commercially available.

Animals. A total of 24 male Sprague-Dawley (SD) rats $(180 \pm 20 \mathrm{~g})$ were purchased from the Experimental Animal Center of Dalian Medical University (Dalian, China). The rats were housed in a controlled temperature of $22 \pm 2^{\circ} \mathrm{C}$ with $60 \pm 5 \%$ relative humidity, a $12 \mathrm{~h}$ light/dark cycle and ad libitum access to water and food; rats were fasted overnight and provided with water prior to surgery. All animal experiments were approved by the ethics committee of Dalian Medical University and performed in accordance with the institutional guidelines.

Rat model of CP. For the purification of rat prostatic protein, male SD rats (240-300 g) were sacrificed and prostate tissue was removed under sterile conditions and washed with saline solution. Prostate tissue was placed into a physiological saline solution containing $0.5 \%$ Triton X-100 and homogenized in an ice-water bath with a glass homogenizer. The homogenized samples were centrifuged $\left(10,000 \times \mathrm{g}\right.$ at $\left.4^{\circ} \mathrm{C}\right)$ for $10 \mathrm{~min}$ and the protein was measured by using a bicinchoninic acid assay (Beijing Solarbio Science and Technology Co., Ltd., Beijing, China) and diluted to $15 \mathrm{mg} / \mathrm{ml}$ in PBS buffer.

Rats were subcutaneously injected with the DPT vaccine $(0.5 \mathrm{ml} / \mathrm{kg})$ at day 0 , and then injected at multipoint sites (right foot pad, left foot pad and base of the tail and shoulders) with a mixture (volume, $1 \mathrm{ml}$ ) of purified rat prostatic protein and Freund's Complete Adjuvant (1:1 ratio) at 0,15 and 30 days. Control rats were injected with same volume of PBS. The rat model of CP was established after 45 days. Rats were randomly divided into the following three groups ( $n=8 /$ group): Control group, rats orally administered with saline for 10 days without $\mathrm{CP}$ induction; $\mathrm{CP}$ group, $\mathrm{CP}$ rats orally administered with saline for 10 days starting on day 45; resveratrol group, CP rats orally administered with resveratrol $(10 \mathrm{mg} / \mathrm{kg}$, dissolved in saline) for 10 days starting on day 45 .

Bladder pressure and volume tests in rats. Rats were anesthetized using an intraperitoneal injection of pentobarbital $(60 \mathrm{mg} / \mathrm{kg}$ ) prior to surgery. Rats were fixed in the operating frame, and the upper edge of the pubic symphysis skin was incised. The bladder was exposed and placed on the incision to avoid the effect of abdominal pressure on the detrusor pressure. Two 24G tubes were inserted into the bladder and fixed, and the bladder was irrigated with saline $(0.4 \mathrm{ml} / \mathrm{min})$ via one of the $24 \mathrm{G}$ tubes. Another tube was connected to a MedLab biological signal acquisition system via a pressure transducer. The maximum capacity of the bladder, residual urine volume and maximum voiding pressure were measured.

Morphological changes. The rats were sacrificed at the end of the bladder pressure and volume test. The prostate was removed, fixed in $10 \%(\mathrm{v} / \mathrm{v})$ neutral formalin for $48 \mathrm{~h}$, then embedded in paraffin and processed using standard histological techniques. sections $(4 \mu \mathrm{m})$ were stained at room temperature with hematoxylin (15 min) and eosin (3 min; $\mathrm{H} \& \mathrm{E}$ ), and examined for morphological changes using light microscope ( 8 fields of view analyzed per sample). The protein expression of tryptase, chymase, TGF- $\beta$, Wnt and $\alpha$-SMA were determined in the samples by western blot analysis or immunohistochemical staining, as described below.

Western blot analysis. Proteins were extracted from rat prostate using a protein extraction kit (Nanjing KeyGen Biotech, Co., Ltd., Nanjing, China) according to the manufacturer's instructions. As a positive control (+) for chymase, a protein sample from the hearts of rats was used. Proteins were measured by using a bicinchoninic acid assay (Beijing Solarbio Science and Technology Co., Ltd., Beijing, China). Proteins (20 $\mu \mathrm{g})$ were resuspended in electrophoresis sample buffer (Beijing Solarbio Science and Technology Co., Ltd.) containing $\beta$-mercaptoethanol and separated by electrophoresis on a pre-cast $10 \%$ SDS-polyacrylamide gel (Bio-Rad Laboratories, Inc., Hercules, CA, USA), followed by electrotransfer to a polyvinylidene difluoride membrane (EMD Millipore, Billerica, MA, USA). Membranes were blocked using 5\% non-fat milk in Tris-buffered saline with $0.1 \%$ Tween-20 (TBST) for $2 \mathrm{~h}$ at $37^{\circ} \mathrm{C} . \beta$-actin served as the loading control. Membranes were incubated overnight at $4^{\circ} \mathrm{C}$ with a 1:1,000 dilution of polyclonal antibodies for tryptase (sc-32889), TGF- $\beta$ (sc-146) and $\beta$-catenin (sc-7199; Santa Cruz Biotechnology, Inc., Dallas, TX, USA) and a 1:1,500 dilution of a monoclonal antibody for chymase (bs-2353R), $\alpha$-SMA (bs-0189R) and $\beta$-actin (bsm-33036M; BIOSS, Beijing, China). Membranes were subsequently washed with TBST and incubated with 1:1,000 secondary antibodies (sc-2004; Santa Cruz Biotechnology, Inc.) for $1 \mathrm{~h}$ at $37^{\circ} \mathrm{C}$. Blots were extensively washed with TBST and exposed to Enhanced Chemiluminescence Plus reagent (Beyotime Institute of Biotechnology), according to the manufacturer's instructions. 


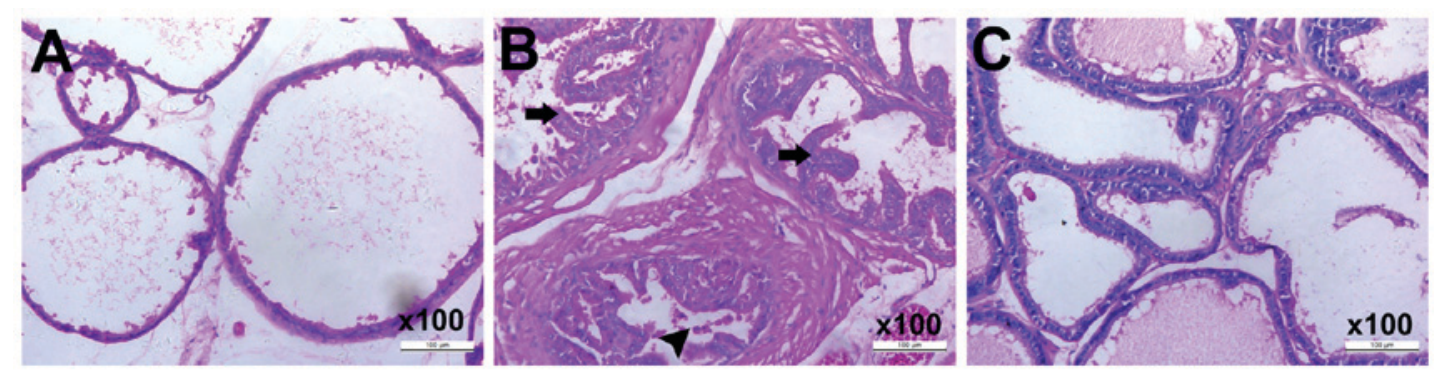

Figure 1. Changes in prostate photomicrographs of CP rats. The prostates were stained with hematoxylin and eosin. (A) Staining of prostate tissue in control group. (B) Leukocyte infiltration (arrowhead), papillary frond protrusion into the gland cavities (arrows) and a notable increase in prostatic epithelial height was observed in the CP group compared with the control group. Gland lumen diameter was also markedly smaller in the CP group. (C) The observed alterations in the $\mathrm{CP}$ group were suppressed in $\mathrm{CP}$ rats treated with resveratrol. Magnification, $\mathrm{x} 100$. CP, chronic prostatitis.

Emitted light was documented using a BioSpectrum-410 multispectral imaging system with a Chemi HR camera 410 (Bio-Rad Laboratories, Inc.). Protein bands were visualized and images were captured under transmitted ultraviolet light. The images (three images per animal) were used for semi-quantitative measurements based on band densitometry. Blots were semi-quantified by densitometric analysis using the Image Lab software version 4.0 (Bio-Rad Laboratories, Inc.).

Immunohistochemical staining. The prostates were fixed in $10 \%(\mathrm{v} / \mathrm{v})$ neutral formalin for $48 \mathrm{~h}$, then embedded in paraffin, Histological sections of rat prostate $(4 \mu \mathrm{m})$ were mounted onto poly-L-lysine-coated slides. Slides were deparaffinized in xylene and rehydrated in graded alcohols. Sections were pretreated with citrate buffer $(0.01 \mathrm{~mol} / \mathrm{l}$ citric acid, $\mathrm{pH} 6.0)$ for $20 \mathrm{~min}$ at $95^{\circ} \mathrm{C}$ and immersed in PBS containing $3 \% \mathrm{H}_{2} \mathrm{O}_{2}$ for $10 \mathrm{~min}$ at room temperature. Sections were exposed to $10 \%$ normal goat serum (streptavidin/peroxidase; SP-9001; ZSGB-BIO, Beijing, China) in PBS for $30 \mathrm{~min}$ at room temperature and incubated at $4^{\circ} \mathrm{C}$ overnight with rabbit polyclonal anti-tryptase or anti-chymase antibodies mentioned above (1:100 dilution; Santa Cruz Biotechnology, Inc.). Sections were rinsed with PBS, incubated with biotinylated goat anti-rabbit IgG (SP-9001; ZSGB-BIO) for $20 \mathrm{~min}$ at room temperature and treated with 3,3-diaminobenzidine chromogen for $5 \mathrm{~min}$ at room temperature. Sections were counterstained under light microscope with hematoxylin (C0107, Beyotime Institute of Biotechnology) for $2 \mathrm{~min}$ at room temperature.

$M C$ assessment. Two independent investigators, who were blinded to the study, assessed MC infiltration. Each examiner analyzed five randomized high-power fields in the tissues following immunohistochemical staining in each group, and analysis was performed at different magnifications. The number and morphology of the MCs were evaluated. The 5 areas with the highest number of MCs were identified at low power (x100), and the number of MCs in the selected fields were counted at a higher power (x400). The number of MCs was recorded as the total number in 5 different areas at high power $(\mathrm{x} 400)$. The location and morphology of tryptase (+) cells was considered while counting. Tryptase (+) cells with a round to oval shape and granular nuclei were considered to be MCs.

Collagen determination. The prostates were fixed in $10 \%$ $(\mathrm{v} / \mathrm{v})$ neutral formalin for $48 \mathrm{~h}$, then embedded in paraffin
Table I. Resveratrol improves overactive bladder in CP rats.

\begin{tabular}{lccc}
\hline Group & $\begin{array}{c}\text { Maximum } \\
\text { capacity, } \mathrm{ml}\end{array}$ & $\begin{array}{c}\text { Maximum voiding } \\
\text { pressure, } \mathrm{cm} \mathrm{H} \mathrm{H}_{2} \mathrm{O}\end{array}$ & $\begin{array}{c}\text { Residual urine } \\
\text { volume, } \mathrm{ml}\end{array}$ \\
\hline Control & $0.51 \pm 0.05$ & $27.24 \pm 1.52$ & $0.17 \pm 0.03$ \\
$\mathrm{CP}$ & $0.94 \pm 0.17^{\mathrm{a}}$ & $35.64 \pm 2.61^{\mathrm{a}}$ & $0.52 \pm 0.03^{\mathrm{a}}$ \\
Resveratrol & $0.72 \pm 0.40^{\mathrm{a}, \mathrm{b}}$ & $30.51 \pm 1.15^{\mathrm{a}, \mathrm{b}}$ & $0.28 \pm 0.05^{\mathrm{a}, \mathrm{b}}$
\end{tabular}

${ }^{\mathrm{a}} \mathrm{P}<0.01$ vs. control group; ${ }^{\mathrm{b}} \mathrm{P}<0.01$ vs. $\mathrm{CP}$ group. $\mathrm{CP}$, chronic prostatitis.

and $4 \mu \mathrm{m}$ thick sections mounted onto poly-L-lysine-coated slides. Picrosirius red staining was performed on serially sectioned tissues. Paraffin-embedded tissues were deparaffinized in xylene, rehydrated in graded alcohols and incubated in a $0.1 \%$ picrosirius red solution for $1 \mathrm{~h}$ at room temperature. Sections were counterstained with hematoxylin for $2 \mathrm{~min}$ at room temperature. The sections were examined under a light microscope.

Statistical analysis. Statistical analysis was performed using SPSS version 13.0 software (SPSS, Inc., Chicago, IL, USA). Data are presented as the mean + standard deviation. Statistically significant differences between two datasets were determined using one-way analysis of variance followed by Tukey's post hoc test. $\mathrm{P}<0.05$ was considered to indicate a statistically significant difference.

\section{Results}

Histological examinations. The extent of inflammatory cell infiltration, acinar alterations and interstitial fibrosis were evaluated following treatment. Compared with the control group (Fig. 1A), the CP model group (Fig. 1B) exhibited severe diffuse chronic inflammation, characterized by leukocyte infiltration and papillary frond protrusion into the gland cavities and a notable increase in prostatic epithelial height. Gland lumen diameter was also markedly smaller in the lateral lobe of the prostate. However, these changes were suppressed in the rats treated with resveratrol (Fig. 1C).

Resveratrol improves the overactive bladder in $C P$ rats. The results of the bladder pressure and volume test revealed that the maximum capacity of the bladder, maximum voiding 

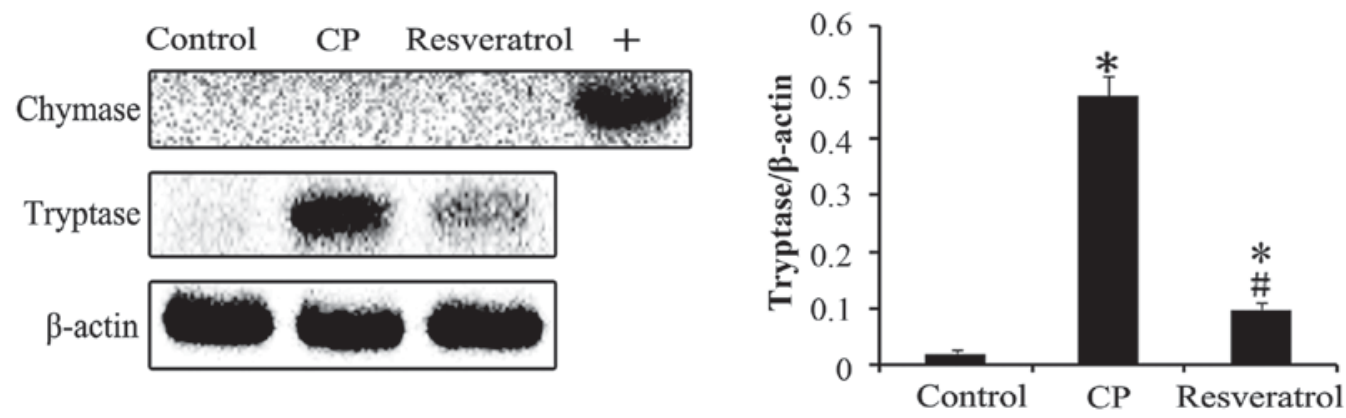

Figure 2. Effect of resveratrol on the expression of tryptase and chymase proteins in the prostates of $\mathrm{CP}$ rats. Compared with the control group, tryptase was significantly upregulated in the $\mathrm{CP}$ group, and the upregulation was significantly suppressed by resveratrol treatment. However, chymase was not detected in any of the groups. As a positive control (+) for chymase, a protein sample from the hearts of rats was used. The bar graph presents the relative expression ratio of tryptase calculated following normalization to $\beta$-actin. Data are presented as the mean \pm standard deviation. ${ }^{*} \mathrm{P}<0.01$ vs. control group; ${ }^{\#} \mathrm{P}<0.01$ vs. $\mathrm{CP}$ group; $\mathrm{n}=8$. $\mathrm{CP}$, chronic prostatitis.
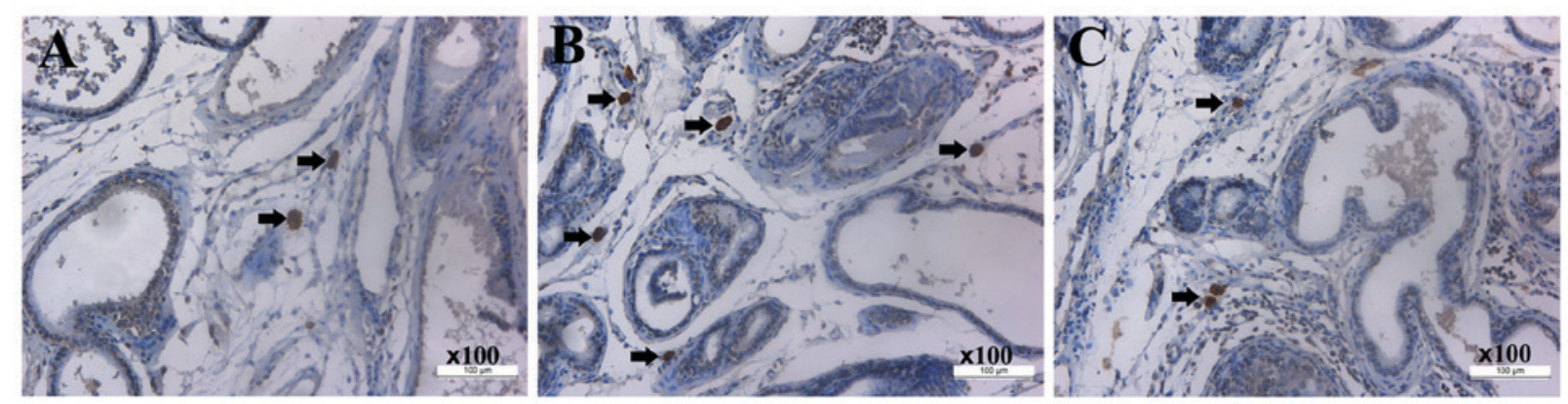

Figure 3. MC infiltration in the prostates. Tissues were stained using immunohistochemistry for tryptase. Tryptase-positive MC (arrows) infiltration occurred around the gland area in the stroma. Representative images of tissue staining in (A) control, (B) CP and (C) resveratrol groups. Magnification, x100. MC, mast cell; $\mathrm{CP}$, chronic prostatitis.

Table II. Mast cell counts using immunohistochemical staining in each group.

\begin{tabular}{lrrc}
\hline & \multicolumn{3}{c}{ Counts using immunohistochemical staining } \\
\cline { 2 - 4 } Groups & Examiner 1 & Examiner 2 & \multicolumn{1}{c}{ Mean } \\
\hline Control & $3.17 \pm 1.17$ & $3.50 \pm 1.05$ & $3.33 \pm 1.07$ \\
CP & $13.50 \pm 1.38$ & $13.67 \pm 1.03$ & $13.58 \pm 1.16^{\mathrm{a}}$ \\
Resveratrol & $8.33 \pm 0.82$ & $8.83 \pm 1.17$ & $8.58 \pm 1.00^{\mathrm{a}, \mathrm{b}}$ \\
\hline
\end{tabular}

${ }^{\mathrm{a}} \mathrm{P}<0.01$ vs. control group; ${ }^{\mathrm{b}} \mathrm{P}<0.01$ vs. CP group. $\mathrm{CP}$, chronic prostatitis

pressure and residual urine volume in the control group were $0.51 \mathrm{ml}, 27.24 \mathrm{~cm} \mathrm{H}_{2} \mathrm{O}$ and $0.17 \mathrm{ml}$, respectively (Table I). The maximum capacity of the bladder, maximum voiding pressure and residual urine volume in the $\mathrm{CP}$ group increased significantly compared with the control group (Table I), which indicated that the CP model was successfully established. Resveratrol treatment of $\mathrm{CP}$ rats markedly reversed the maximum capacity, maximum voiding pressure and residual urine volume of the bladder compared with the $\mathrm{CP}$ group (Table I). These results indicate that resveratrol effectively improved an overactive bladder in $\mathrm{CP}$ rats.

Resveratrol reduces tryptase (+) $M C$ infiltration in $C P$ rats, but chymase (+) MCs are not detected. MCs have an essential role in fibrosis, and their phenotype and function during resveratrol treatment for $\mathrm{CP}$ was investigated in the present study. Rat prostates were immediately acquired following sacrifice for western blot analysis and immunohistochemical assays. Western blotting revealed that the expression levels of tryptase protein in the prostate of the $\mathrm{CP}$ group was increased significantly compared with the control group (Fig. 2). However, resveratrol treatment of $\mathrm{CP}$ rats significantly decreased expression of this protein compared with the CP group (Fig. 2). The expression of chymase was not detected using western blotting in any of the groups (Fig. 2). Immunohistochemical staining indicated that, compared with the control group (Fig. 3A and Table II), the number of tryptase (+) MCs was significantly higher in the CP group (Fig. 3B and Table II). Resveratrol treatment of $\mathrm{CP}$ rats resulted in a significant reduction in $\mathrm{MC}$ number in prostate tissue (Fig. 3C and Table II). Chymase (+) MCs were not detected using immunohistochemistry in the prostates from all groups.

Resveratrol inhibits the activity of TGF- $\beta$ and the Wnt/ $\beta$-catenin signaling pathway. TGF- $\beta$ is a profibrotic cytokine that induces macrophage and fibroblast proliferation via the induction of other growth factors. Western blot analysis revealed an increased expression level of TGF- $\beta$ in the $\mathrm{CP}$ group compared with the control group, which was significantly decreased following resveratrol treatment (Fig. 4). The Wnt/ $\beta$-catenin signaling pathway is vital in 

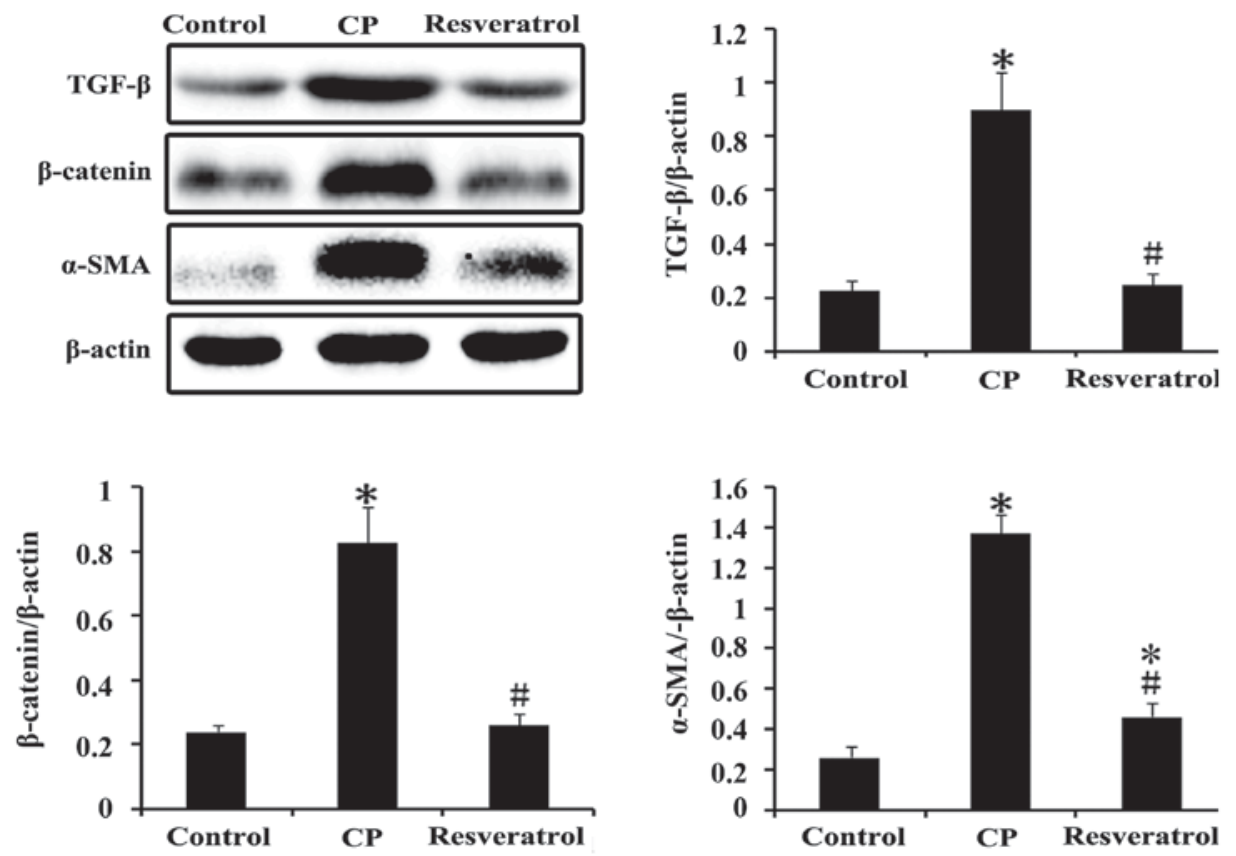

Figure 4. Effect of resveratrol on protein expression levels of $\alpha$-SMA, TGF- $\beta$ and $\beta$-catenin in the prostates of CP rats. Compared with the control group, the expression of the proteins was significantly upregulated in the $\mathrm{CP}$ group. However, the upregulation was significantly suppressed by resveratrol treatment. The bar graph presents the relative expression ratio of each protein calculated following normalization to $\beta$-actin. Data are presented as the mean \pm standard deviation. ${ }^{*} \mathrm{P}<0.01$ vs. control group; ${ }^{*} \mathrm{P}<0.01$ vs. CP group; $\mathrm{n}=8$. $\alpha$-SMA, $\alpha$-smooth muscle actin; TGF- $\beta$, transforming growth factor- $\beta ; \mathrm{CP}$, chronic prostatitis
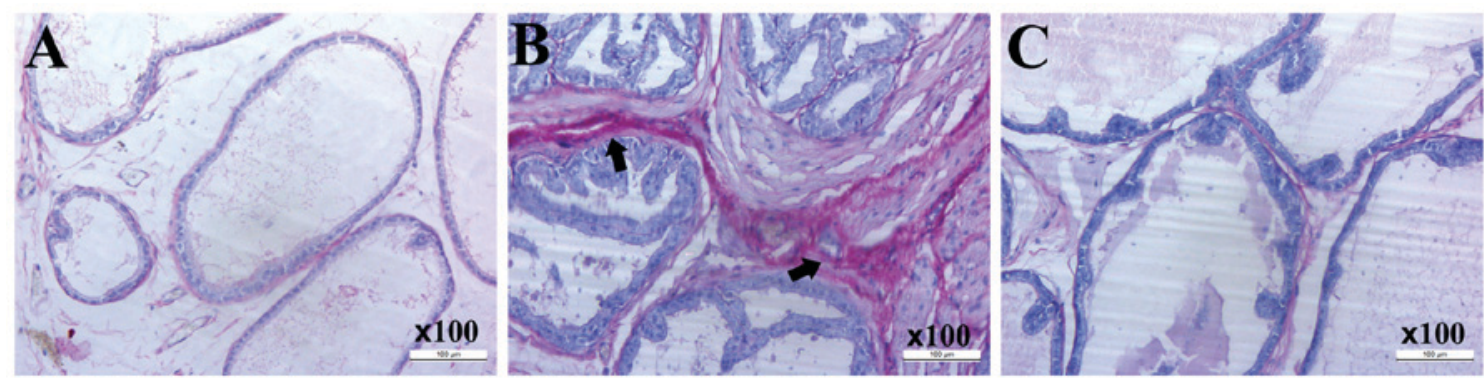

Figure 5. Effect of resveratrol on collagen accumulation in the prostates of $\mathrm{CP}$ rats. Tissues were stained for collagen with picrosirius red. (A) Picrosirius red staining of prostate tissues in the control group. (B) Increased collagen content (arrows) was observed in the CP group compared with the control group. (C) Collagen content in CP rats was reduced following resveratrol treatment. Magnification, x100. CP, chronic prostatitis.

TGF- $\beta$-induced fibrosis. An increase in $\beta$-catenin and $\alpha$-SMA expression was observed in CP tissues compared with the control group, and resveratrol treatment of $\mathrm{CP}$ rats significantly reduced the expression of $\beta$-catenin and $\alpha$-SMA (Fig. 4).

Resveratrol reduces collagen accumulation in CP tissues. Picrosirius red staining indicated that, compared with the control group (Fig. 5A), there was a higher collagen content in the $\mathrm{CP}$ group (Fig. 5B), which was decreased in $\mathrm{CP}$ rats following resveratrol treatment (Fig. 5C).

\section{Discussion}

Ma et al (3) reported that prostatic fibrosis was associated with LUTS; however, the underlying mechanism of LUTS promotion was not investigated. Chronic prostatitis is a common disease in urology and voiding dysfunction is the primary clinical manifestation. The presence of urinary tract obstruction was revealed in patients with CP following urodynamic examination (2). The maximum capacity of the bladder is assessed as a parameter of storage function, and the residual urine volume and maximum voiding pressure are evaluated as parameters of voiding function. In the present study, the maximum capacity of the bladder, residual urine volume and maximum voiding pressure in rats in the $\mathrm{CP}$ group were increased significantly compared with the control group. However, following treatment with resveratrol, these changes were suppressed.

We hypothesized that increased collagen accumulation in the prostate during $\mathrm{CP}$ progression is a consequence of fibrosis, which increases tissue stiffness. Periurethral tissue fibrosis may reduce the flexibility and capacity of the prostatic urethra to expand to accommodate urinary flow, which may lead to symptoms associated with obstruction. Fibrosis is a connective tissue remodeling process that is characterized by the activation and accumulation of myofibroblasts (24), of which $\alpha$-SMA is a marker. The present study revealed that $\alpha$-SMA expression was significantly higher in the $\mathrm{CP}$ group compared 
with the control group, and resveratrol treatment of $\mathrm{CP}$ rats decreased $\alpha$-SMA expression. Picrosirius red staining demonstrated markedly increased collagen content in the CP group compared with the control group, and the maximum capacity of the bladder, residual urine volume and maximum voiding pressure in the $\mathrm{CP}$ group increased significantly compared with the control group. Furthermore, H\&E staining in CP rats revealed high leukocyte infiltration and papillary fronds that protruded into the gland cavities, and there was a notable increase in prostatic epithelial height and a smaller gland lumen diameter. However, resveratrol treatment suppressed these alterations.

MCs are important effector cells in tissue fibrosis that express the serine proteases, tryptase and chymase, which are the most abundant proteins in MCs. Tryptase and chymase have been reported to stimulate the proliferation of fibroblasts, induce collagen synthesis in fibroblast cultures and induce collagen fibril formation $(25,26)$. However, limited information is known regarding the involvement of these proteases in CP. The results of the present study demonstrated that the number of tryptase (+) MCs was significantly higher in the $\mathrm{CP}$ group compared with the control group. Resveratrol administration to $\mathrm{CP}$ rats significantly reduced the number of tryptase (+) MC in prostate tissues. However, chymase (+) MCs were not detected in the prostates from all groups which indicated that mast cell chymase was not involved in prostatitis progression. As previously reported (27), the primary fibrotic role of tryptase in the heart is the induction of MC degranulation, which induces the release of chymase and promotes fibrosis. However, Beghdadi et al (28) reported that MC protease 4 , the functional counterpart of human chymase, exhibited antifibrotic potential in acutely induced obstructive nephropathy. The present study indicated that tryptase may individually induce prostate fibrosis in CP in the absence of chymase. The molecular phenotypic heterogeneity of MC makes them attractive therapeutic targets in various diseases $(29,30)$. The tryptase ${ }^{+} /$chymase $^{-}$MC may serve a critical role in CP. Therefore, the fibrosis-inducing mechanism, which MC is dependent on, is complex and further studies are required.

The profibrotic effects of MCs are closely associated with TGF- $\beta$. Studies have demonstrated that MC numbers and staining intensity were associated with increased TGF- $\beta$ production and interstitial fibrosis $(12,13)$. TGF- $\beta$-induced fibrosis process primarily occurs via the Smad pathway, which serves a critical role in transmitting TGF- $\beta$ signals. The Wnt/ $\beta$-catenin signaling pathway is also important in the genesis of fibrosis in various organ systems. The $\mathrm{Wnt} / \beta$-catenin signaling pathway was reported to be a downstream regulatory pathway of the TGF- $\beta$ pathway (31). TGF- $\beta$ activates canonical Wnt signaling, which is implicated in the profibrotic effects of TGF- $\beta$ (32). The Wnt/ $\beta$-catenin signaling pathway is vital in TGF- $\beta$-induced fibrosis (14). Wnt signaling has been reported to skew TGF- $\beta$ signaling towards dominant signaling via the Smad pathway (33). Therefore, interactions of Wnt with TGF- $\beta /$ Smad signaling requires further investigation in different situations. CP tissues in the present study exhibited increased TGF- $\beta$ and $\beta$-catenin expression, which was reduced in the resveratrol treatment group. Control tissue sections exhibited limited positive expression.
Resveratrol (15), a botanical compound that is derived primarily from the skins of red grapes, has been extensively employed in traditional medicine and also as a dietary supplement. A previous study demonstrated that resveratrol may inhibit tissue fibrosis in various organs (34). In addition, resveratrol repressed and reversed prostate fibroblast to myofibroblast phenoconversion in vitro (18). Consistent with these findings, the results of the present study demonstrated that resveratrol treatment markedly inhibited the overactivity of MCs, the TGF- $\beta / \mathrm{Wnt} / \beta$-catenin pathway and tissue fibrosis in the $\mathrm{CP}$ tissues, therefore improving voiding dysfunction in $\mathrm{CP}$ rats.

In conclusion, the progression of urinary dysfunction in $\mathrm{CP}$ may be induced by fibrosis in the prostate. Notably, in the present study, tissues from $\mathrm{CP}$ rats revealed increased $\mathrm{MC}$ infiltration and an upregulated activity of the TGF- $\beta / \mathrm{Wnt} / \beta$-catenin pathway, which were reduced following resveratrol treatment. The results of the present study demonstrated the antifibrotic effect of resveratrol. Therefore, resveratrol may be considered as a candidate for the treatment of CP. MCs are not only important for the periurethral tissue fibrosis in $\mathrm{CP}$, but are also a potential target for therapy.

\section{Acknowledgements}

The study of the present study was technically supported by the College of Pharmacy of Dalian Medical University and the Second Affiliated Hospital of Dalian Medical University (Dalian, China). The study was funded by a project supported by the Natural Science Foundation of Liaoning province (grant no. 201601229).

\section{References}

1. Tyagi P, Kashyap M, Pore S, Wang Z and Yoshimura N: MP25-07 prostatic inflammation evokes upergulation of neurotrophins in sensory ganglia: Possible contribution to dysfunctional voiding. J Urol 193, e287, 2015

2. Liao LM, Shi BY and Liang CQ: Ambulatory urodynamic monitoring of external urethral sphincter behavior in chronic prostatitis patients. Asian J Androl 1: 215-217, 1999.

3. Ma J, Gharaee-Kermani M, Kunju L, Hollingsworth JM, Adler J, Arruda EM and Macoska JA: Prostatic fibrosis is associated with lower urinary tract symptoms. J Urolo 188: 1375-1381, 2012.

4. Karsdal MA, Genovese F, Madsen EA, Manon-Jensen T and Schuppan D: Collagen and tissue turnover as a function of age: Implications for fibrosis. J Hepatol 64: 103-109, 2016.

5. Fabre V, Wu H, PondTor S, Coutinho H, Acosta L, Jiz M, Olveda R, Cheng L, White ES, Jarilla B, et al: Tissue inhibitor of matrix-metalloprotease 1 predicts risk of hepatic fibrosis in human schistosoma japonicum infection. J Infect Dis 203: 707-714, 2011.

6. Trujillo KA, Heaphy CM, Mai M, Vargas KM, Jones AC, Vo P Butler KS, Joste NE, Bisoffi M and Griffith JK: Markers of fibrosis and epithelial to mesenchymal transition demonstrate field cancerization in histologically normal tissue adjacent to breast tumors. Int J Cancer 129, 1310-1321, 2011.

7. Silver RB: Role of mast cells in renal fibrosis. Kidney Int 84: 214, 2013.

8. Cruse $\mathrm{G}$ and Bradding P: Mast cells in airway diseases and interstitial lung disease. Eur J Pharma 778: 125-138, 2016.

9. Gaca MDA, Arthur MJP and Benyon RC: Mast cell protease and stem cell factor expression in rat liver fibrosis. J Hepatol 28: 81, 1998.

10. Yadav A, Desai RS, Bhuta BA, Singh JS, Mehta R and Nehete AP: Altered immunohistochemical expression of mast cell tryptase and chymase in the pathogenesis of oral submucous fibrosis and malignant transformation of the overlying epithelium. PLoS One 9: e98719, 2014. 
11. Zerr P, Palumbo-Zerr K, Huang J, Tomcik M, Sumova B Distler O, Schett G and Distler JH: Sirt1 regulates canonical TGF- $(\beta)$ signalling to control fibroblast activation and tissue fibrosis. Ann Rheum Dis 75: 226-233, 2016.

12. Summers SA, Gan PY, Dewage L, Ma FT, Ooi JD, O'Sullivan KM Nikolic-Paterson DJ, Kitching AR and Holdsworth SR: Mast cell activation and degranulation promotes renal fibrosis in experimental unilateral ureteric obstruction. Kidney Int 82: 676-685, 2012.

13. Jeong DH, Lee GP, Jeong WI, Do SH, Yang HJ, Yuan DW, Park HY, Kim KJ and Jeong KS: Alterations of mast cells and TGF-betal on the silymarin treatment for CCI(4)-induced hepatic fibrosis. World J Gastroenterol 11: $1141-1148,2005$

14. Akhmetshina A, Palumbo K, Dees C, Bergmann C, Venalis P, Zerr P, Horn A, Kireva T, Beyer C, Zwerina J, et al: Activation of canonical Wnt signalling is required for TGF- $\beta$-mediated fibrosis. Nat Commun 3: 735, 2012.

15. Torres P, Poveda A, Jimenez-Barbero J, Ballesteros A and Plou FJ: Regioselective lipase-catalyzed synthesis of 3-o-acyl derivatives of resveratrol and study of their antioxidant properties. J Agric Food Chem 58: 807-813, 2010.

16. Sharma S, Anjaneyulu M, Kulkarni SK and Chopra K Resveratrol, a polyphenolic phytoalexin, attenuates diabetic nephropathy in rats. Pharmacology 76: 69-75, 2006.

17. Csiszar A: Anti-inflammatory effects of resveratrol: Possible role in prevention of age-related cardiovascular disease. Ann N Y Acad Sci 1215, 117-122, 2011.

18. Gharaee-Kermani M, Moore BB and Macoska JA: Resveratrol-mediated repression and reversion of prostatic myofibroblast phenoconversion. PLoS One 11: e 0158357, 2016.

19. He Y, Zeng H, Yu Y, Zhang J, Zeng X, Gong F, Duan X, Liu Q and Yang B: Resveratrol improved the progression of chronic prostatitis via the downregulation of c-kit/SCF by activating Sirt1. J Agric Food Chem 65: 5668-5673, 2017.

20. He Y, Zeng HZ, Yu Y, Zhang JS, Duan X, Zeng XN, Gong FT, Liu Q and Yang B: Resveratrol improves prostate fibrosis during progression of urinary dysfunction in chronic prostatitis. Environ Toxicol Pharmacol 54: 120-124, 2017.

21. Wong L, Done JD, Schaeffer AJ and Thumbikat P: Experimental autoimmune prostatitis induces microglial activation in the spinal cord. Prostate 75: 50-59, 2015.

22. Zheng T, Wang R, Zhang TB, Jia DH, Wang CL, Sun Y and Zhang WX: AB036. Effects and its potential mechanisms of Cox-2 inhibitors on ejaculation latency of rat with experimental autoimmune prostatitis. Transl Androl Urol 5 (Suppl 1): AB036, 2016.

23. Rivero VE, Motrich RD, Maccioni $M$ and Riera $C M$ : Autoimmune etiology in chronic prostatitis syndrome: An advance in the understanding of this pathology. Crit Rev Immunol 27: 33-46, 2007.
24. Fraga F, Sturny M, Ruoccolo R, Santos R, Silva R and Stergiopulos N: 139 Angiotensin-(1-7) inhibits cavernosal fibrosis via attenuation of fibroblast differentiation to myofibroblast. J Sex Med 14 (Suppl 1): S39, 2017.

25. Abe M, Kurosawa M, Ishikawa O, Miyachi Y and Kido H: Mast cell tryptase stimulates both human dermal fibroblast proliferation and type I collagen production. Clin Exp Allergy 28 1509-1517, 1998

26. Kofford MW, Schwartz LB, Schechter NM, Yager DR, Diegelmann RF and Graham MF: Cleavage of type I procollagen by human mast cell chymase initiates collagen fibril formation and generates a unique carboxyl-terminal propeptide. J Biol Chem 272: 7127-7131, 1997

27. Li J, Jubair S, Levick SP and Janicki JS: The autocrine role of tryptase in pressure overload-induced mast cell activation, chymase release and cardiac fibrosis. IJC Metabolic and Endocr 10: 16-23, 2016.

28. Beghdadi W, Madjene LC, Claver J, Pejler G, Beaudoin L, Lehuen A, Daugas E and Blank U: Mast cell chymase protects against renal fibrosis in murine unilateral ureteral obstruction. Kidney Int 84: 317-326, 2013.

29. Globa T, Saptefrţi L, Ceauşu RA, Gaje P, Cimpean AM and Raica M: Mast cell phenotype in benign and malignant tumors of the prostate. Pol J Pathol 65: 147-153, 2014.

30. Qi L, Song W, Liu Z, Zhao X, Cao W and Sun B: Wnt3a promotes the vasculogenic mimicry formation of colon cancer via wnt $/ \beta$-catenin signaling. Int J Mol Sci 16: 18564-18579, 2015.

31. Conidi A, van den Berghe V and Huylebroeck D: Aptamers and their potential to selectively target aspects of EGF, Wnt $/ \beta$-catenin and TGF $\beta$-smad family signaling. Int J Mol Sci 14: 6690-6719, 2013

32. Pons M, Ali L, Beghdadi W, Danelli L, Alison M, Madjène LC, Calvo J, Claver J, Vibhushan S, Åbrink M, et al: Mast cells and MCPT4 chymase promote renal impairment after partial ureteral obstruction. Front Immunol 8: 450, 2017.

33. van den Bosch $\mathrm{MH}$, Blom AB, van Lent PL, van Beuningen HM, Blaney Davidson EN, van der Kraan PM and van den Berg WB: Canonical Wnt signaling skews TGF-beta signaling in chondrocytes towards signaling via ALK1 and Smad 1/5/8. Cell Signal 26: 951-958, 2014.

34. Xiao Z, Chen C, Meng T, Zhang W and Zhou Q: Resveratrol attenuates renal injury and fibrosis by inhibiting transforming growth factor- $\beta$ pathway on matrix metalloproteinase 7. Exp Biol Med (Maywood) 241: 140-146, 2016. International (CC BY-NC-ND 4.0) License. 